\title{
A COUNTEREXAMPLE TO A PROBLEM OF SZ.-NAGY1
}

S. R. FOGUEL

The purpose of this note is to construct an operator, in a Hilbert space, with uniformly bounded powers, which is not similar to a contraction. (An operator $P$ is a contraction if $\|P\| \leqq 1$.) This will furnish a negative answer to a problem raised in [1].

Let $H$ be a Hilbert space and $T$ an operator such that $\left\|T^{n}\right\|$ $\leqq M(n=1,2, \cdots)$. Define

$$
H_{0}(T)=\left\{x \mid \text { weak } \lim T^{n} x=0\right\}, \quad H_{1}(T)=H_{0}\left(T^{*}\right)^{\perp} .
$$

We proved in [2, Theorem 3.1] that if $P$ is a contraction then $H_{0}(P)=H_{0}\left(P^{*}\right)$. Let $T=S P S^{-1}$ where $\|P\| \leqq 1$, then:

$$
H_{0}(T)=\left\{x \mid P^{n} S^{-1} x \stackrel{\omega}{\rightarrow} 0\right\}=\left\{x \mid S^{-1} x \in H_{0}(P)\right\}=S\left(H_{0}(P)\right)
$$

and

$$
\begin{aligned}
H_{1}(T) & =H_{0}\left(T^{*}\right)^{\perp}=\left[S^{*-1}\left(H_{0}\left(P^{*}\right)\right)\right]^{\perp}=\left\{x \mid S^{-1} x \perp H_{0}\left(P^{*}\right)\right\} \\
& =S\left(H_{1}(P)\right) .
\end{aligned}
$$

But $H_{1}(P) \perp H_{0}(P)$ and thus $H_{0}(T) \cap H_{1}(T)=0$.

We will construct an operator, with uniformly bounded iterates, for which $H_{0} \cap H_{1} \neq 0$, and thus the operator is not similar to a contraction.

Let $H=K \oplus L$, where $K$ is generated by the orthonormal sequence $\left\{e_{i}\right\}$ and $L$ by the orthonormal sequence $\left\{f_{i}\right\}$. Let $\left\{n_{k}\right\}$ be a subsequence of the integers which is "sparse" in the sense that:

$$
n_{k+1}-n_{k}>2 n_{k}
$$

(e.g., $n_{k}=4^{k}$ ).

Notation. Integers in the sequence $\left\{n_{k}\right\}$ will be denoted by $\alpha, \beta, \cdots$. Integers outside the sequence $\left\{n_{k}\right\}$ will be denoted by $a, b, \cdots$. The letters $i, j, k \ldots$ will stand for integers which might be in or out of the exceptional sequence $\left\{n_{k}\right\}$.

Definition. Let the operator $T$ be defined by the linear extension of

$$
T e_{1}=0, \quad T e_{i}=e_{i-1}, \quad i \geqq 2,
$$

and

Received by the editors December 21, 1962 and, in revised form, May 2, 1963.

1 The research in this document has been sponsored in part by the Air Force Office of Scientific Research, OAR, through the European Office. Aerospace Research, United States Air Force. 


$$
T f_{a}=f_{a+1}, \quad T f_{\alpha}=e_{\alpha}+f_{\alpha+1} .
$$

LEMMA 1. For each $j$ and $h$

$$
T^{j} f_{h}=f_{h+j}+\epsilon(j, h) e_{i(j, h)}
$$

where:

a. If no element of the sequence $n_{k}$ is in $[h, h+j)$ then $\epsilon(j, h)=0$.

b. Let $\alpha$ be the largest element of $\left\{n_{k}\right\}$ with $h \leqq \alpha<h+j$ then:

$$
\begin{array}{ll}
\epsilon(j, h)=0 & \text { if } 2 \alpha<j+h, \\
\epsilon(j, h)=1 & \text { if } 2 \alpha \geqq j+h
\end{array}
$$

and

$$
i(j, h)=2 \alpha-j-h+1 .
$$

Proof. Let us prove by induction on $j$. For $j=1$ the proof is clear. Assume the lemma holds for $j$. Now if no element of $\left\{n_{k}\right\}$ is in $[h, h+j)$ then there are two possibilities:

1. If $h+j \notin\left\{n_{k}\right\}$ then

$$
T^{j+1} f_{h}=T\left(T^{j} f_{h}\right)=T f_{h+j}=f_{j+h+1} \text { and } \epsilon(j+1, h)=0 .
$$

2. If $h+j=\alpha \in\left\{n_{k}\right\}$ then

$$
T^{j+1} f_{h}=T f_{\alpha}=f_{\alpha+1}+e_{\alpha}=f_{h+j+1}+e_{2 \alpha-j-h} .
$$

On the other hand, let $\alpha$ be the largest element of $\left\{n_{k}\right\}$ with $h \leqq \alpha$ $<h+j$. Again there are two possibilities:

1. If $h+j=\beta \in\left\{n_{k}\right\}$ then $h+j=\beta>2 \alpha$ and $\epsilon(j, h)=0$ hence

$$
T^{j+1} f_{h}=T f_{j+h}=T f_{\beta}=f_{j+h+1}+e_{j+h}=f_{j+h+1}+e_{2 \beta-j-h} .
$$

2. If $h+j \notin\left\{n_{k}\right\}$ then

$$
\begin{aligned}
T^{j+1} f_{h} & =T\left(T^{j} f_{h}\right)=T\left(f_{j+h}+\epsilon(j, h) e_{i(j, h)}\right) \\
& =f_{j+h+1}+\epsilon(j, h) T e_{i(j, h)} .
\end{aligned}
$$

Now if $2 \alpha<j+h$ then $\epsilon(j, h)=0$ and $\epsilon(j+1, h)=0$ since $\alpha$ is the largest element of $\left\{n_{k}\right\}$ in $[h, j+h+1)$ and $2 \alpha<j+h+1$. However if $2 \alpha \geqq j+h$ then

$$
T^{j+1} f_{h}=f_{j+h+1}+T e_{2 \alpha-j-h+1}=f_{j+h+1}+e_{2 \alpha-j-h},
$$

where $e_{0}=0$.

Thus $\epsilon(j+1, h)=1$ if $2 \alpha \geqq j+h+1$ and then

$$
i(j+1, h)=2 \alpha-j-h .
$$

while $\epsilon(j+1, h)=0$ if $2 \alpha=j+h$. 
Lemma 2. Let $j$ be given and $h_{1} \neq h_{2}$. If $\epsilon\left(j, h_{1}\right)=\epsilon\left(j, h_{2}\right)=1$ then $i\left(j, h_{1}\right) \neq i\left(j, h_{2}\right)$.

Proof. Let $\alpha_{1}$ and $\alpha_{2}$ be the largest elements of $\left\{n_{k}\right\}$ in $\left[h_{1}, j+h_{1}\right)$ and $\left[h_{2}, j+h_{2}\right)$ respectively. If $\alpha_{1}=\alpha_{2}$ then

$$
i\left(j, h_{1}\right)=2 \alpha_{1}-j-h_{1}+1 \neq 2 \alpha_{1}-j-h_{2}+1=i\left(j, h_{2}\right) .
$$

If $\alpha_{2}>\alpha_{1}$ then

$$
2 \alpha_{1}>i+h_{1}>j
$$

since $\epsilon\left(j, h_{1}\right)=1$. Thus

$$
i\left(j, h_{2}\right)=2 \alpha_{2}-j-h_{2}+1 \geqq \alpha_{2}-j \geqq \alpha_{2}-2 \alpha_{1}>\alpha_{1}
$$

but

$$
\alpha_{1} \geqq 2 \alpha_{1}-j-h_{1}=i\left(j, h_{1}\right),
$$

since $\alpha_{1}<j+h_{1}$ by its definition.

LEMMA 3. $\left\|T^{j}\right\| \leqq 2$.

Proof. Let $y=\sum a_{h} f_{h} \in L$; then

$$
\left\|T^{j} y\right\|^{2}=\left\|\sum a_{h} f_{j+h}\right\|^{2}+\left\|\sum a_{h} \epsilon(j, h) e_{i(j, h)}\right\|^{2}
$$

and since the indices $i(j, h)$ are different, for different values of $h$, we get

$$
\left\|T^{j} y\right\|^{2} \leqq 2 \sum\left|a_{h}\right|^{2}=2\|y\|^{2} .
$$

Let $z \in H$; then $z=x+y$ where $x \in K$ and $y \in L$. Then

$$
\begin{aligned}
\left\|T^{j} z\right\|^{2} & \leqq\left(\left\|T^{j} x\right\|+\left\|T^{j} y\right\|\right)^{2} \leqq(\|x\|+\sqrt{ } 2\|y\|)^{2} \\
& \leqq 2(\|x\|+\|y\|)^{2} \leqq 4\left(\|x\|^{2}+\|y\|^{2}\right)=4\|z\|^{2} .
\end{aligned}
$$

LEMMA 4. The vector $e_{1}$ belongs to both $H_{0}(T)$ and $H_{1}(T)$.

Proof. Clearly $e_{1} \in H_{0}(T)$. Now $T^{2 n_{k}-1} f_{1}=f_{2 n_{k}}+e_{1} \rightarrow^{\omega} e_{1}$. Thus if $z \in H_{0}\left(T^{*}\right)$ then

$$
\left(e_{1}, z\right)=\lim \left(T^{2 n_{k}-1} f_{1}, z\right)=\lim \left(f_{1}, T^{* 2 n_{k}-1} z\right)=0,
$$

since weak $\lim T^{*_{n}} z=0$. Hence $e_{1} \in H_{0}\left(T^{*}\right)^{\perp}=H_{1}(T)$.

\section{BIBLIOGRAPHY}

1. B. Sz.-Nagy, Completely continuous operators with uniformly bounded iterates, Magyar Tud. Akad. Mat. Kutat6 Int. Közl. 4 (1959), 89-93.

2. S. R. Foguel, Powers of a contraction in Hilbert space, Pacific J. Math. 13 (1963), 551-562.

HEBREW UNIVERSITY, JERUSALEM 